\title{
Excitatory and inhibitory consequences of explicitly unpaired and truly random conditioning procedures on heart rate in rats
}

\author{
CHRISTOPHER L. CUNNINGHAM, ROBERT D. FITZGERALD \\ and DAVID L. FRANCISCO \\ University of Oregon Health Sciences Center, Portland, Oregon 97201
}

\begin{abstract}
A comparison was made of heart-rate $(\mathrm{HR})$ responses of restrained rats to CSs that were part of an explicitly unpaired or a truly random control procedure. Subsequent to these procedures, an assessment was made of the relative capacities of these CSs to affect an established HR CR in a combined-cue paradigm and to impede the development of a HR CR in a reversal-conditioning situation. The principal findings were (1) that the explicitly unpaired and truly random CSs generated HR responses of opposite direction, i.e., HR acceleration vs. HR deceleration, respectively, and (2) that conditioning of a decelerative HR CR to the CS that had earlier been employed in the explicitly unpaired procedure was retarded compared to what was obtained to the truly random CS. The two CSs did not have reliably different effects in the combined-cue test. It was suggested that the truly random CS may have produced both associative and nonassociative influences on HR. It was hypothesized further than the explicitly unpaired CS may have acquired the capacity to function as a conditioned inhibiting stimulus.
\end{abstract}

The question of what temporal relationship between presentations of the CS and the US may constitute the most appropriate control procedure for nonassociative factors during excitatory classical conditioning has been considered in detail in recent theoretical treatments of the conditioning process, (e.g., Prokasy, 1965; Rescorla, 1967). Based on a contingency view of this process, Rescorla (1967) argued that the commonly used explicitly unpaired procedure may not be adequate because of the negative contingency that is produced by presenting the US in the absence of the CS. This was thought to promote an inhibitory response tendency, resulting possibly in an underestimation of nonassociative influences on responding. As an alternative control arrangement, Rescorla proposed that the CS and US be presented on a completely random basis, thereby eliminating any contingent or correlative relationship between the two events. In the absence of either positive or negative contingencies, the random $\mathrm{CS}$, even though occasionally paired with the US by chance, was predicted to yield a measure of baseline

This research was supported by National Science Foundation Grant BMS74-18542, by an Oregon Heart Association grant, and in part by a U.S. Public Health Service grant, 5 S01 RR05412. Christopher L. Cunningham was a National Institute of General Medical Sciences predoctoral trainee (GM01495-07) and is now at Yale University. David L. Francisco was a predoctoral trainee on a National Institute on Alcohol Abuse and Alcoholism grant (5 T01-AA00033-03). Requests for reprints should be sent to Robert D. Fitzgerald, Department of Medical Psychology, University of Oregon Health Sciences Center, 3181 S. W. Sam Jackson Park Road, Portland, Oregon 97201. performance relatively unaffected by either excitatory or inhibitory factors.

Support for the notion that an explicitly unpaired CS acquires inhibitory tendencies has been provided by classical-to-instrumental transfer experiments showing that the rate of ongoing avoidance responding (e.g., Bull \& Overmier, 1968; Rescorla, 1966) and the level of suppression in a CER paradigm (e.g., Rescorla, 1969a) were reduced in the presence of the CS. At the same time, however, other studies have revealed what appeared to be substantial excitatory effects to a randomly presented CS as measured in a CER situation (Benedict \& Ayres, 1972; Kremer \& Kamin, 1971; Quinsey, 1971). These effects, which seemed to be dependent upon chance CS-US pairings occurring early in the random sequence (Benedict $\&$ Ayres, 1972), suggest that the truly random control may result in an inflated estimate of nonassociative responding during classical conditioning.

In spite of the relevance of the control-group issue to the study of classical conditioning, few reports have been made of the extent to which inhibitory and excitatory phenomena that have been demonstrated in transfer studies may occur in traditional classical conditioning investigations involving the direct measurement of the conditioned response. Moreover, those studies that have been carried out have provided mixed results. In a series of experiments examining the skin-conductance reaction in humans (summarized in Furedy, Poulos, \& Schiffmann, 1975), no differences were found between subjects receiving an explicitly unpaired procedure and those given a truly random treatment. In contrast, a study 
in which a within-subjects design was employed provided some evidence that skin-conductance responding in humans was less to an explicitly unpaired CS than to a truly random CS (Prokasy, Williams, Kumpfer, Lee, \& Jensen, 1973). Although this reduction in responding would be consistent with the view that the explicitly unpaired CS had acquired inhibitory capacities, specific tests to examine this possibility (cf. Hearst, 1972; Rescorla, 1969b) were not included as part of the Prokasy et al. study, nor were they carried out in the other skinconductance experiments.

Several investigations have been reported in which a test was made of the effects of backward US-CS pairings-a regime which may qualify as a special case of an explicitly unpaired procedure (Rescorla, 1966b) - on subsequent conditioning of the eyelid and nictitating membrane responses in rabbits (Plotkin \& Oakley, 1975; Siegel \& Domjan, 1971, 1974). The results of these studies showed that acquisition of the conditioned response was markedly retarded to a CS that had a history of backward conditioning. However, in one of the experiments, a truly random procedure also retarded acquisition performance (Siegel \& Domjan, 1971), and in another, an essentially explicitly unpaired procedure failed to have any effect on later acquisition behavior (Plotkin \& Oakley, 1975). There was no evidence of response suppression to any of the "inhibitory" CSs during exposure to unpaired presentations with the US. In view of the extremely low baseline or spontaneous level of eyelid responding demonstrated by rabbits in classical conditioning situations (cf. Gormezano, 1966), the failure to find suppression is perhaps not surprising.

The current experiment had two objectives. The first was to determine whether there was a difference in the heart-rate (HR) responses of restrained rats to explicitly unpaired and truly random CSs. In this regard, it was felt that ongoing cardiac activity might provide an appropriate "above-zero"' baseline against which the potentially divergent responseeliciting tendencies of these CSs could be evaluated. The second objective was to assess the relative capacities of the unpaired and random CSs to modify an established conditioned HR response in a summation or combined-cue test and to influence the development of a conditioned $\mathrm{HR}$ response in a newlearning-retardation or reversal-conditioning test. Both of these test procedures have been used to identify inhibitory stimuli (Hearst, 1972; Rescorla, 1969b).

\section{METHOD}

\section{Subjects}

Thirty-two naive female albino (Wistar) rats, ranging 240-295 g in weight and 100-130 days old, were used. They were purchased from the Department of Animal Care of the University of Oregon
Health Sciences Center and individually caged with free access to food and water under a normal 12-h light-dark cycle. Experimental sessions occurred during the light cycle.

\section{Apparatus}

The rats were restrained in an inverted-U-shaped acrylic animal holder made by $\mathrm{E} \& \mathrm{M}$ Instrument Company. Adjustable guillotine-type inserts at the ends of the holder were positioned to hold the rats securely. The holder was placed on the floor of a $25.4 \mathrm{~cm}$ wide $\times 30.5 \mathrm{~cm}$ long $\times 40.6 \mathrm{~cm}$ high aluminum enclosure located inside an Industrial Acoustics sound-attenuated chamber. A $60-\mathrm{W}$ houselight recessed in the ceiling illuminated the chamber, and masking noise was delivered to a speaker mounted on the back wall of the chamber $(70 \mathrm{~dB}$, re .0002 dyne/ $\mathrm{cm}^{2}$ ). Two $10.2-\mathrm{cm}$ speakers were mounted on the ceiling of the aluminum enclosure.

Pairs of rats were trained concurrently in separate identical chambers with trials alternated between the rats. The electrocardiogram (EKG) was recorded on a Grass polygraph from 20-ga hypodermic needles located on either side of the rat's thoracic cavity. The number of heart beats occurring in consecutive time intervals within a trial was tabulated automatically by means of an on-line recording system similar to that described by Fitzgerald, Vardaris, and Teyler (1968). Basically, the system consisted of a lever-type microswitch mounted on a Plexiglas plate directly above the EKG polygraph pen, with the arm of the switch connected to the tip of the pen. The position of the switch was adjusted so that it was actuated by the $R$ wave of the QRS complex. The number of $\mathbf{R}$ waves was accumulated and punched on a high-speed paper-tape perforator.

The two CSs were either a $1-\mathrm{kHz}$ or a $5-\mathrm{kHz}$ tone. Each tone was presented for $6.1 \mathrm{sec}$ through one of the speakers mounted in the conditioning box at a sound pressure level of $80 \mathrm{~dB}( \pm 2 \mathrm{~dB}$ re $.0002 \mathrm{dyne} / \mathrm{cm}^{2}$ ). The US was a .1 -sec train of $100-\mathrm{V} \mathrm{dc} .5-\mathrm{msec}$ pulses at a frequency of $50 \mathrm{~Hz}$ produced by a Massey Dickinson constant energy shocker and delivered to the base of the rat's tail through the heads of No. 6 machine screws that were held in place approximately $2-3 \mathrm{~cm}$ apart with small pieces of rubber tubing.

The CSs and US were started automatically by film-tape programmers. Stimulus events and HR counting intervals were timed by solid-state logic modules.

\section{Procedure}

The study was conducted in six successive phases and involved two groups of rats $(n=16$ each) that were treated identically in all but the third phase. During the first phase, both groups were given 12 presentations of each of the two CSs in a counterbalanced random order at intervals of 60,90 , or $120 \sec (M=90)$. The US was not presented during this phase. In Phase 2, both groups received 24 conditioning trials, with one of the CSs $(C S+)$ being paired with the US at an interstimulus interval of $6 \mathrm{sec}$. The intertrial intervals (ITIs) were 120,150 , or $180 \mathrm{sec}(\mathbf{M}=150)$.

Both groups received 90 presentations of the other CS (the "unpaired" CS) and US during Phase 3. However, during this phase the groups differed in terms of the regularity of the temporal relation between the CS and the US. Rats in the explicitly unpaired group received either the CS alone or the US alone in a randomized order at intervals of 120,150 , or $180 \mathrm{sec}(M=150)$. No more than three CSs or three USs were allowed to occur in a row. Rats in the truly random group received the same sequence of CSs as those in the explicitly unpaired group; however, US onset was programmed randomly with respect to CS onset. This was done by dividing Phase 3 into 450 60-sec intervals and fixing the probability that the US would occur in a given interval at $90 /$ $450=.2$. Thus, the probability of US occurrence during the CS and the 60 -sec interval following CS onset was equal to the probability of the US during all other 60 -sec intervals (i.e., Pr [US/CS] $=\operatorname{Pr}[\mathrm{US} / \overline{\mathrm{CS}}]=.2$ ). The location of the US within a given 60-sec interval was determined randomly. This sequence resulted in approximately six shocks every $30 \mathrm{~min}$ and roughly equated 
the truly random group for the temporal distribution of shocks received by the explicitly unpaired group.

Phase 4 consisted of four additional reinforced CS + trials, identical to those given in Phase 2. Phase 5 was a combined-cue test. On each of 12 trials, CS + and the "unpaired" CS were presented simultaneously for $6.1 \mathrm{sec}$ and the US was not presented. The final phase was reversal conditioning, in which the CS that had been "unpaired" during Phase 3 was paired with shock 24 times at a CS-US interval of $6 \mathrm{sec}$. The ITI during these last phases averaged $150 \mathrm{sec}$.

The six experimental phases were distributed across 3 consecutive days. Each day began with $15 \mathrm{~min}$ of adaptation to the restraining device. The first day consisted of the pretest CS-alone trials of Phase 1, the conditioning trials of Phase 2 and 30 of the "unpaired" trials of Phase 3. The administration of an additional 44 "unpaired" trials comprised Day 2. On Day 3, the remaining 16 "unpaired" trials were given, followed in sequence by the reconditioning trials of Phase 4 , the combined-cue trials of Phase 5, and the reversal-conditioning trials of Phase 6. For half of the animals in each group, the $1-\mathrm{kHz}$ tone was $\mathrm{CS}+$ and the $5-\mathrm{kHz}$ tone was the "unpaired" CS. The roles of these stimuli were reversed for the remaining animals.

Heart-rate responses were recorded during the 6-sec immediately preceding the onset of each CS (pre-CS period) and during three consecutive 2-sec intervals following CS onset. These scores were converted to beats-per-minute (bpm). Difference scores (CS rate minus pre-CS rate) were used to index the response to each stimulus.

\section{RESULTS}

The data presented in the figures below were collapsed across the $1-5-\mathrm{kHz}$ frequency dimension of the CSs. However, frequency of the CSs was included as a factor in all of the analyses of variance that are reported.

Figure 1 shows the HR reactions of the explicitly unpaired and truly random groups averaged over the final four pretest CS-alone trials of Phase 1 and over the 24 conditioning trials of Phase 2. Inspection of the left side of the figure reveals that the overall direction of the HR orienting response on the pretest trials was decelerative and that, with the possible exception of the first counting period, the magnitudes of the responses of the groups were similar. In both cases, the cardiodecelerations were larger at the beginning than at the end of the $C S, F(2,56)=3.93$, $\mathrm{p}<.05$. The mean $\mathrm{HR}$ response to the $5-\mathrm{kHz}$ tone $(-9.6 \mathrm{bpm})$ was significantly larger than that occurring to the $1-\mathrm{kHz}$ tone $(-3.5 \mathrm{bpm}), F(1,28)$ $=13.01, \mathrm{p}<.001$. There were no significant differences between the groups during this phase of the study.

The right side of the figure indicates that the direction of the conditioned HR response was cardiodeceleration, with the magnitude of the response increasing progressively over the three counting periods of the CS-US interval. A 2 by 2 by 3 (Explicitly Unpaired vs. Truly Random by Frequency of CS by Counting Periods) analysis of variance on these data provided a significant effect of counting periods, $F(2,56)=23.36, p<.001$, and a significant Counting Periods by Frequency of CS interaction, $F(2,56)$

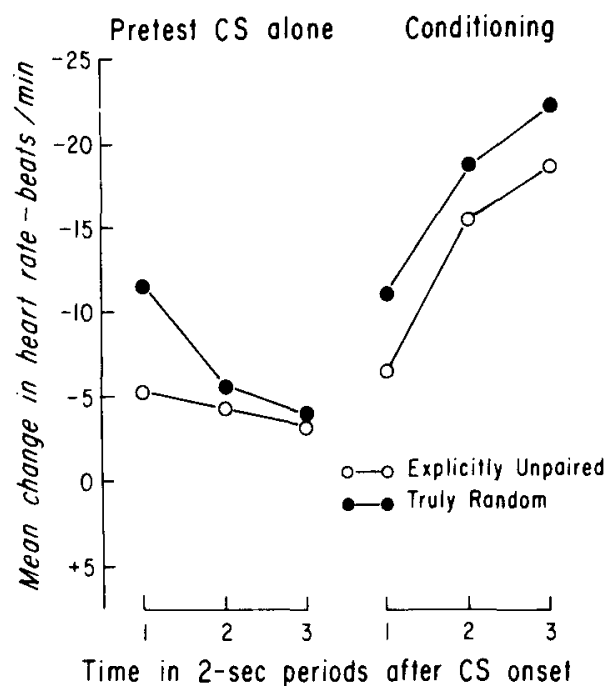

Figure 1. Mean CS minus pre-CS heart-rate changes of the explicitly unpaired and truly random groups on the final 4 pretest CS-alone trials (Phase 1) and on the 24 conditioning trials (Phase 2).

$=4.78, \mathrm{p}<.05$. This interaction was due to a slightly greater deceleration to the onset of the $1-\mathrm{kHz}$ than to the $5-\mathrm{kHz} \mathrm{CS}$, with the reactions then converging to the same level during the final two counting periods of the CSs.

To minimize the direct effects of the US on the HR reactions of the truly random group to the CS during the third, "unpaired" phase of the experiment, those trials (total $=27$ ) in which the US occurred either during the CS or within a 1 -min period prior to the onset of the CS were eliminated. To make the groups comparable, the same trials were discarded for the explicitly unpaired group. Figure 2 shows the HR reactions of the two groups during the three counting

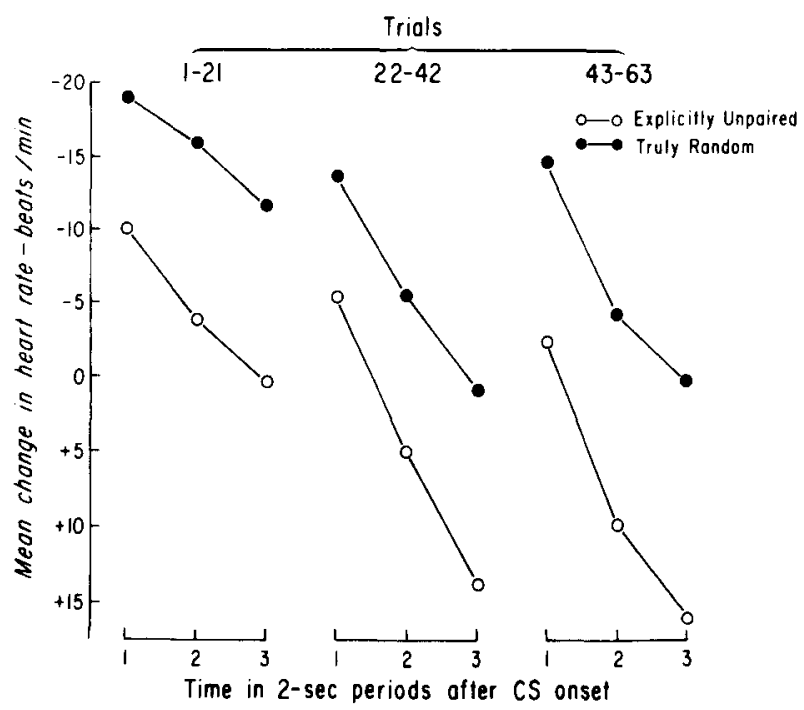

Figure 2. Mean CS minus pre-CS heart-rate changes of the explicitly unpaired and truly random groups in successive blocks of 21 "unpaired"' trials (Phase 3). 
periods of the "unpaired" CS on the remaining 63 trials averaged over successive blocks of 21 trials each. In general, the overall direction of the HR response shown by the truly random group was a monophasic deceleration that decreased in magnitude, especially during the second and third counting periods, over trial blocks. On the last trial block, HR deceleration in this group was restricted mainly to CS onset. In the case of the explicitly unpaired group, the direction of the HR response changed from cardiodeceleration on the initial block of trials to a biphasic deceleration-acceleration on the middle block of trials and then to what was basically a monophasic acceleration on the final block of trials. Separate $t$ tests established that the HR accelerations in the third counting period of the middle trial block and in the second and third counting periods of the final trial block were significantly different from zero ( $p<.01$, df $=15$, in each case). The deceleration in the first counting period was significantly different from zero during the middle block of trials, $t(15)=$ $2.59, \mathrm{p}<.01$, but not during the final block. A fourway analysis of variance of the data shown in Figure 2 revealed that the overall difference between the two groups was significant, $F(1,28)=13.46$, $\mathrm{p}<.001$. There was also a significant effect of trials, $F(2,56)=24.35, p<.001$, a significant effect of counting periods, $F(2,56)=68.29, p<.001$, a significant Counting periods by Trials interaction, $F(4,112)=7.89, p<.001$, and a significant Groups by Frequency of CS by Counting Periods by Trials interaction, $F(4,112)=3.77, p<.01$. The latter interaction was due to the fact that the cardioaccelerations shown by the explicitly unpaired group developed sooner to the $5-\mathrm{kHz}$ tone than to the $1-\mathrm{kHz}$ tone. An examination of the 27 eliminated trials of the third phase averaged in three blocks of nine trials each demonstrated HR changes that were highly similar to those shown in Figure 2. Moreover, an analysis of variance on these trials produced outcomes comparable to those reported above for Phase 3.

Figure 3 depicts the HR responses of the two groups averaged over the four reacquisition trials of Phase 4 and over three successive blocks of four combined-cue trials during Phase 5 . It will be recalled that, in Phase 5, CS + and the "unpaired" CS were presented together in the absence of the US. Considering first the reacquisition data shown in the far left of the figure, it is evident that the overall magnitudes of the HR responses of both groups were somewhat smaller than those obtained during original conditioning. Furthermore, the topography of the CR in the explicitly unpaired group did not appear to recover fully during reacquisition. However, an analysis of variance indicated that $C R$ topographies of the two groups did not differ significantly from each other during reacquisition, with

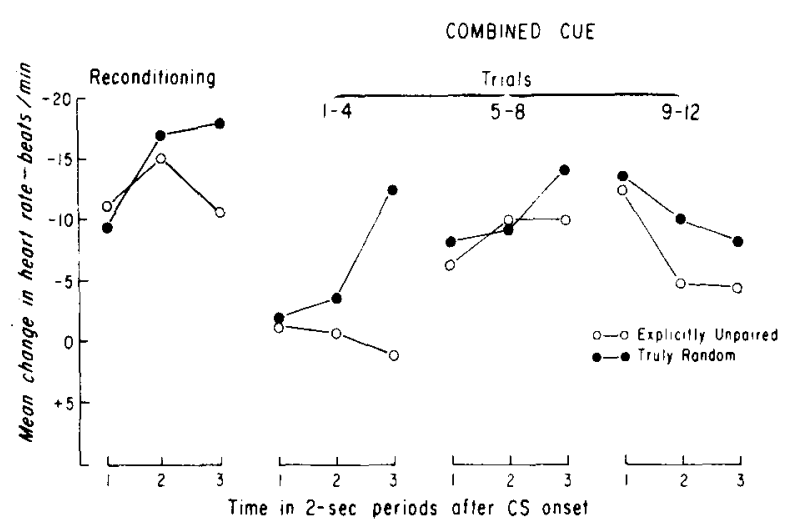

Figure 3. Mean CS minus pre-CS heart-rate changes of the explicitly unpaired and truly random groups on reconditioning (Phase 4) and combined-cue trials (Phase 5).

both groups still showing a significant overall change in $\mathrm{HR}$ across the three counting periods, $F(2,56)=$ $3.17, \mathrm{p}<.05$.

Turning now to the results of the combined-cue test displayed in the right side of Figure 3, there is visual evidence that during the first block of trials the responses of the explicitly unpaired group in the second and third counting periods were depressed relative to those of the truly random group. However, an analysis of variance on the data in just the first block provided no significant differences bebetween the groups. A 2 by 2 by 3 by 3 analysis of variance carried out on all of the combined-cue data shown in Figure 3 provided a significant Counting Periods by Trials interaction, $F(4,112)=3.69$, $p<$ .01 , reflecting the change in the topography of the HR reactions of the combined groups over trials.

The results obtained during the reversal phase in which the previously "unpaired" CS was now paired with the US are presented in blocks of six trials in Figure 4. It is evident from an inspection of this figure that there were major differences between the groups in terms of the direction and topography of the $H R$ responses. In the case of the truly random group, HR decelerations on the first block of trials

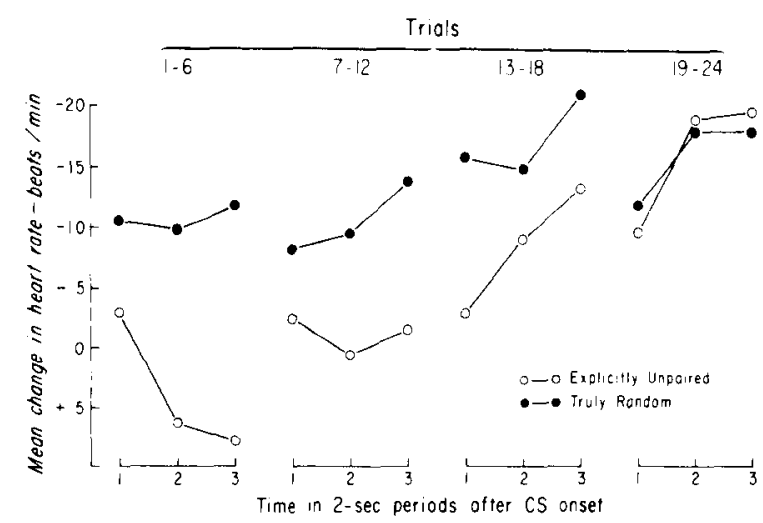

Figure 4. Mean CS minus pre-CS heart-rate changes of the explicitly unpaired and truly random groups in successive sixtrial blocks of reversal conditioning (Phase 6). 
were comparable in each of the three periods of the CS-US interval. As conditioning progressed, cardiodecelerations became larger in the second and third counting periods, with the topography of the CR on the final block of trials being very similar to that which occurred during original conditioning to CS + . On the other hand, the HR reaction of the explicitly unpaired group on the initial block of reversal trials resembled the accelerative response elicited by the same CS at the end of the "unpaired" phase of the study. In this case, HR deceleration during the first counting period of the CS was followed by HR accelerations during the second and third counting period. Both of these accelerations were significantly different from zero according to separate $t$ tests ( $p<.025$, df $=15$, in each case), whereas the deceleration was not. On subsequent trial blocks, there was a gradual transformation of the response with $H R$ accelerations in the second and third counting intervals giving way to HR decelerations. By the end of conditioning, the form of the CR of the explicitly unpaired group matched that of the truly random group in that cardiodecelerations were maximal in the last two counting periods of the CS-US interval.

A four-way analysis of variance performed on these results provided a significant effect of trials, $F(3,84)=14.78, p<.01$, a significant Groups by Trials interaction, $F(3,84)=4.06, p<.05$, a significant Counting Periods by Trials interaction, $F(6,168)$ $=5.24, \mathrm{p}<.01$, and a significant Groups by Counting Periods by Trials interaction, $F(6,168)$ $=2.22, \mathrm{p}<.05$, demonstrating the reliability of the differential change in the topographies of the HR responses of the two groups over conditioning trials. In a second analysis, carried out on just the HR responses during the third counting period, the overall difference between the groups was significant, $F(1,28)=5.02, p<.05$, as was the trials effect, $F(3,84)=16.60, p<.01$, and the Groups by Trials interaction, $F(3,84)=5.19, \mathrm{p}<.05$.

Between-group comparisons were made of pre-CS or baseline HR averaged across the same trial blocks as those plotted for the HR reactions to the CSs in the previous figures. Although the mean baseline HRs of the explicitly unpaired group were consistently below those of the truly-random group during each of the six phases of the study, the differences were significant only during the last three phases. The individual means in bpm for the truly random and explicitly unpaired groups were, respectively: Phase 1 CS alone, 454 vs. 429; Phase 2 conditioning, 423 vs. 404; Phase 3 "unpaired," 428 vs. 412 ; Phase 4 reconditioning, 457 vs. $415, F(1,28)=10.74, p<.01$; Phase 5 combined cue, 453 vs. $426, F(1,28)=4.61$, $\mathrm{p}<.05$; Phase 6 reversal conditioning, 429 vs. 401 , $F(1,28)=4.92, p<.05$. During the latter phase, the HR levels of both groups decreased similarly across trials, $F(3,84)=38.70, p<.001$. A separate analysis on the eliminated trials of the third, "unpaired" phase revealed that the mean baseline HR of the truly random group (444 bpm) was significantly elevated above that of the explicitly unpaired group (411 bpm), $\mathrm{F}(1,28)=9.35, \mathrm{p}<.01$.

\section{DISCUSSION}

The main findings of the present study were that: (1) the explicitly unpaired and truly random procedures produced $H R$ responses of opposite directions to the CSs on the "unpaired" trials; (2) subsequent to the "unpaired" trials, development of a conditioned $\mathrm{HR}$ response to the explicitly unpaired CS was retarded relative to that shown to the truly random CS; and (3) the explicitly unpaired and truly random CSs failed to have significant differential effects on an established HR CR in a combined-cue paradigm.

The observed difference in the direction of the $H R$ responses of the explicitly unpaired and truly random groups developed over the course of the "unpaired" phase. Early in the phase, both groups displayed predominantly monophasic cardiodecelerations that matched the decelerative direction of the CR. Because the "unpaired" trials were given after the CR had been established, it seems likely that these initial decelerations may have been partly due to stimulus generalization between CS + and the "unpaired" CSs. With further exposure to the "unpaired" regime, the decelerative response in the explicitly unpaired group changed to a biphasic deceleration acceleration and then to a monophasic acceleration. On the other hand, the response of the truly random group persisted throughout the "unpaired" trials as a monophasic deceleration.

These findings show, at least under the conditions of the present experiment, that explicitly unpaired and truly random procedures do not provide equivalent base-level measures of HR against which a conditioning paradigm might be compared. This is especially true if a large number of trials is given. Too few trials could account for the fact that cardioaccelerations were not found in prior studies using explicitly unpaired control procedures (e.g., Fitzgerald \& Martin, 1971; Fitzgerald, Martin, \& O'Brien, 1973). In none of these studies were more than 40 presentations of the CS and US administered. In one investigation in which a relatively small HR acceleration was reported in restrained rats, the explicitly unpaired condition consisted of $160 \mathrm{CS}$ and $80 \mathrm{CS}$ presentations distributed across 5 consecutive days (Holdstock \& Scwartzbaum, 1965). An additional possibility is that the cardioacceleration in the present experiment may have been dependent upon the fact that the explicitly unpaired animals received paired excitatory conditioning trials prior to the "unpaired" trials. In the earlier studies failing to show HR accelerations, a between-groups design 
was employed in which the explicitly unpaired group was not exposed to a specific excitatory training paradigm.

One factor that may have contributed to the durability of the CR-like cardiodeceleration shown by the truly random group in the current experiment was the chance CS-US pairings that were generated by the truly random protocol during the "unpaired" session. Evidence that such pairings early in the random sequence may produce conditioning effects has been reported in studies of conditioned suppression (e.g., Benedict \& Ayres, 1972). In the present experiment, there were 12 occasions for each subject in the truly random group, in which the US was presented within a 30 -sec period following CS onset. These trials were approximately equally distributed across successive blocks of 30 presentations of the CS and US (i.e., four per block), with the first trial occurring on the 14th presentation of the CS and US. While it may not be unreasonable to assume that some conditioning could have occurred on these trials as well as on longer CS-US interval trials (Roberts \& Young, 1971), two aspects of the decelerative response in the truly random group argue against its being viewed solely in associative terms.

First, the overall magnitude of the reaction decreased rather than increased across the "unpaired" session. This was most evident during the middle and last counting periods of the CS. Second, and perhaps more important, was the fact that the dynamics or topography of the response was unlike that of the CR to $\mathrm{CS}+$. Thus, HR deceleration to the random CS was maximal during the first $2 \mathrm{sec}$ of the stimulus whereas $\mathrm{HR}$ deceleration to $\mathrm{CS}+$ achieved its peak level during the final $2 \mathrm{sec}$ of the stimulus. This inhibition-of-delaylike topography of the HR response to the CS + has been a characteristic feature of the HR CRs of restrained rats (Fitzgerald \& Martin, 1971; Fitzgerald, Martin, \& Hoffman, 1975; Fitzgerald, Martin, \& O'Brien, 1973) and may offer a useful criterion for distinguishing between associative and nonassociative HR changes during conditioning. On the other hand, maximum cardiodecelerative responses to CS onset similar to those that occurred to the random CS have been found using training paradigms that failed to generate reliable conditioning (Fitzgerald \& Martin, 1971). Fitzgerald and Martin's suggestion that these relatively short-latency reactions may represent sensitized or augmented components of the decelerative orienting response to the CS might also apply to the cardiodecelerations that were obtained in the truly random group. This suggestion could account for the observation that, except for being larger in magnitude, the $\mathrm{HR}$ response to the truly random CS was basically the same as that found on the pretest CS-alone trials.

Possibly the most parsimonious interpretation of the HR acceleration produced by the explicitly unpaired CS during the "unpaired" session involves the concept of conditioned inhibition (Hearst, 1972; Rescorla, 1969b). Rescorla (1969b) defined conditioned inhibitors as those stimuli which, through a particular relationship with the US (e.g., explicitly unpaired), acquired the capacity to control a response tendency opposite to that occurring to a stimulus having a history of excitatory conditioning. He also proposed that the inhibitory and excitatory tendencies should be established using the same US. The explicitly unpaired CS in the present experiment appeared to meet all of these requirements in that (1) the accelerative response to the CS developed over the course of the unpaired session in a manner analogous to a learned reaction, (2) the direction of the response was opposite to the excitatory decelerative CR to CS +, and (3) the CS came to elicit HR acceleration on the basis of a US that earlier supported HR deceleration to $\mathrm{CS}+$. Thus, antagonistic HR responses were produced in the same subjects by simply rearranging the temporal relationship between the respective CSs and the US. A positive relationship in which the US was always presented in close temporal proximity to the CS resulted in cardiodeceleration. On the other hand, a negative relationship that prevented the US from appearing closer than 2 min to the CS eventually led to cardioacceleration.

It should be noted, however, that this evidence for inhibition is unlike the previously obtained in classical-to-instrumental transfer studies (e.g., Bull \& Overmier, 1968; Rescorla, 1969a). In those studies, the explicitly unpaired stimulus was labeled as inhibitory on the basis of its capacity to reduce behavior instilled by excitatory procedures, whereas in the current study the stimulus elicited a response which was, in fact, exactly opposite to that established during excitatory conditioning. The presence of a specific response of this type suggests that it may be worthwhile, in studies of conditioned inhibition, to use experimental procedures that permit the measurement of potentially relevant behavior on a trial-by-trial basis during inhibitory training.

A contrasting conceptual framework to that offered by conditioned inhibition would be to postulate that the cardioacceleration exhibited by the explicitly unpaired group was prompted simply by a decrease in conditioned excitation (cf. Hearst, 1972). It might be speculated that the unsignaled shocks during the "unpaired" phase established a persistent excitatory state of conditioned HR deceleration to the contextual or background cues in the experimental situation which would be measured as a suppression in the level of pre-CS or baseline HR. If this conditioning occurred, the delivery of the "unpaired" CS may have triggered a reduction in the magnitude of the excitatory decelerative $C R$ which, on the basis of the difference-score measure of HR employed, would necessarily generate an 
accelerative HR change to the CS. While difficult to discount, this hypothesis was not supported by finding that baseline HR of the explicitly unpaired group in the "unpaired" phase was comparable to what it was in the paired-excitatory-conditioning phases. With the exception of reconditioning (Phase 4), this was also the case for the truly random group. Presumably, "unpaired" as compared to paired trials should have resulted in lower baseline levels, since relatively more excitatory conditioning should have accrued to background cues with "unpaired" trials.

More generally, baseline HR by itself cannot be used as an explanation of the opposing directions of the HR reactions within the explicitly unpaired group or between the explicitly unpaired and truly random groups. Thus, almost identical baseline HR levels in the explicitly unpaired group were associated with HR deceleration during excitatory conditioning and $H R$ acceleration during inhibitory conditioning. In addition, baseline HR in the two groups was not reliably different during the "unpaired" phase when HR reactions of opposite direction occurred. Finally, the two groups had significantly different baseline levels during Phase 4, 5, and 6 and yet their HR reactions during Phases 4 and 5 and during the latter portion of Phase 6 were similar. In previous work, we have found no evidence that direction or magnitude of the restrained rat's HR response to a CS was determined by the absolute level of HR just prior to the delivery of the CS (e.g., Fitzgerald \& Martin, 1971; Fitzgerald \& Teyler, 1970). It is typical of restrained rats receiving aversive classical conditioning to show decreases in baseline HR once shock is experienced, but regardless of the degree of slowing HR decelerates even further when the CS is presented.

Finding that the HR CR was more difficult to establish in the explicitly unpaired group than in the truly random group during the reversalconditioning phase of the experiment lends additional credibility to the suggestion that the explicitly unpaired CS possessed inhibitory properties. At the beginning of reversal conditioning, the explicitly unpaired stimulus elicited a predominantly accelerative HR response which was similar in topography to the one that occurred on the terminal "unpaired" trials. The fact that this response was found to be more or less intact in a new situation following 4 reconditioning and 12 combined-cue trials indicates that it was more than a transient change in HR, detectable only while the explicitly unpaired procedure was being administered. The occurrence of this response also argues against passive nonresponding (cf. Hearst, 1972) as an explanation of the impeded development of the CR. Following the initial acceleration, the direction of the reversal-conditioning reaction was gradually transformed to HR deceleration with a topography that matched that of the HR CR to CS +. In contrast, the response of the truly random group was decelerative throughout reversal conditioning and achieved a CR-like topography earlier than that displayed by the explicitly unpaired group. Other examples of decremented conditioning to a CS that was previously negatively correlated with a US have been provided for conditioned salivation (Konorski, 1967; Szwekjowska, 1959) and for conditioned suppression in a CER situation (Hammond, 1968; Rescorla, 1969a).

Alternative conceptions to that of inhibition have been outlined to account for decrements in behavior to stimuli being tested for inhibitory tendencies in combined-cue and reversal-conditioning paradigms (Hearst, 1972; Rescorla, 1969b). One of these centers on the notion that behavioral decrements may occur because prior "inhibitory" training leads the subject to be less attentive to the test stimulus. Although decreased attention could account for the failure of the explicitly unpaired stimulus to impair performance in the combined-cue test in the current study, it does not seem applicable with respect to the retarded development of the CR that occurred to this stimulus during reversal conditioning. Thus, the fact that the explicitly unpaired stimulus was accompanied by substantial HR activity, not only on the "unpaired" trials but also on the reversalconditioning trials themselves, provides rather convincing evidence that the stimulus was "noticed."

A second alternative to inhibition attributes decrements in behavior in the presence of a test stimulus to the prior acquisition of a response to the stimulus that interferes with the behavior being measured (Hearst, 1972). On the basis of this viewpoint, the retarded reversal-conditioning performance of the explicitly unpaired group would have been due to the accelerative reaction set up to the explicitly unpaired CS during the "unpaired" trials competing with the subsequent development of the decelerative CR to that CS. Little or no interference would have been expected in the truly random group as the direction of their HR response on the "unpaired" trials matched that of the CR. A competing-response theory also seems supported by the observation that the form of the HR response for the explicitly unpaired group during the first block of combined-cue testing can be predicted quite accurately by averaging the terminal level of responding to the "unpaired" stimulus and the level of responding to $\mathrm{CS}+$ during reconditioning. This is not the case for the random group.

Taken together, then, the inhibition-test results of the present experiment appear to fit comfortably into a competing-response framework. However, that the direction of the HR reaction per se may not have been the critical factor determining responding on the inhibition tests is indicated by recent results 
reported by Fitzgerald and Hoffman (1976). They found that acquisition of a decelerative $H R C R$ in restrained rats was actually facilitated to a CS that elicited HR accelerations following 50 preconditioning presentations of the CS. Although the magnitude of the accelerative response in the Fitzgerald and Hoffman (1976) study was somewhat smaller than that obtained here to the explicitly unpaired CS, their results nevertheless suggest that a competing response explanation of the current findings would have to emphasize something other than a peripheralresponse mechanism.

One approach that might be worth considering would be to view cardioacceleration to the explicitly unpaired CS as reflecting activity of a central located state that had the capacity to impede conditioning of cardiodeceleration. Conceivably, this state could have involved increased sympathetic output, which would be antagonistic to the increased vagal firing that is known to be primarily responsible for HR CRs in the restrained rats (Fitzgerald, Martin, \& O'Brien, 1973). Finding that $\mathrm{HR}$ acceleration to the explicitly unpaired CS was due principally to an increase in sympathetic activity rather than to a simple reduction in vagal output would provide further evidence against the reduced-excitation explanation of the cardioacceleration mentioned earlier.

\section{REFERENCES}

Benedict, J. O., \& AYres, J. J. B. Factors affecting conditioning in the truly random control procedure in the rat. Journal of Comparative and Physiological Psychology, 1972, 78, 323-330.

Bull, J. A., III, \& Overmier, J. B. Additive and subtractive properties of excitation and inhibition. Joumal of Comparative and Physiological Psychology, 1968, 66, 511-514.

Fitzgerald, R. D., \& Hoffman, J. W. Classically conditioned heart rate in rats following preconditioning exposure to the CS. Animal Learning and Behavior, 1976, 4, 58-60.

Fitzgerald, R. D., \& Martin, G. K. Heart rate conditioning in rats as a function of interstimulus interval. Psychological Reports. 1971, 29. 1103-1110.

Fitzgerald, R. D., Martin, G. K., \& Hoffman, J. W. Classical conditioning of heart rate in rats using direct vagal stimulation as a US. Physiology and Behavior, 1975, 14, 449.456.

Fitzgerald, R. D., Martin, G. K., \& O'Brien, J. H. Influence of vagal activity on classically conditioned heart rate in rats. Journal of Comparative and Physiological Psychology, 1973, 83, 485-491.

Fitzgerald, R. D., \& Teyler, T. J. Trace and delayed heart rate conditioning in rats as a function of US intensity. Journal of Comparative and Physiological Psychology, 1970, 70, 242.253.

Fitzgerald, R. D., Vardaris, R. M., \& Teyler, T. J. An on-line method for measuring heart rate in conditioning experiments. Psychophysiology, 1968, 4, 352-353.
Furedy, J. J., Poulos, C. X., \& Schiffman, K. Contingency theory and classical autonomic excitatory and inhibitory conditioning: Some problems of assessment and interpretation. Psychophysiology, 1975, 12, 98-105.

Gormezano, I. Classical conditioning. In J. B. Sidowski (Ed.), Experimental methods and instrumentation in psychology. New York: McGraw-Hill, 1966.

Hammond, L. J. Retardation of fear acquisition when the CS has previously been inhibitory. Journal of Comparative and Physiological Psychology, 1968, 66, 756-759.

Hearst, E. Some persistent problems in the analysis of conditioned inhibition. In R. A. Boakes \& M. S. Halliday (Eds.), Inhibition and learning. New York: Academic Press. 1972.

Holdstock, T. L., \& Schwartzbaum, J. S. Classical conditioning of heart rate and galvanic skin response in the rat. Psychophysiology, 1965, 2, 25-38.

KONORSKI, J. Integrative activity of the brain. Chicago: University of Chicago Press, 1967.

Kremer, E. F., \& Kamin, L. J. Truly random control procedure: Associative or nonassociative effects in rats. Journal of Comparative and Physiological Psychology, 1971. 74. 203-210.

Plotxin, H. C., \& OAKley, D. A. Backward conditioning in the rabbit (Oryctolagus cuniculus). Joumal of Comparative and Physiological Psychology, 1975, 88, 586-590.

Prokasy, W. F. Classical eyelid conditioning: Experimenter operations, task demands, and response shaping. In W. F. Prokasy (Ed.), Classical conditioning. New York: AppletonCentury-Crofts, 1965.

Prokasy, W. F., Williams, W. C., Kumpfer, K. I., Lee, W. Y., \& JENSEN, W. R. Differential SCR conditioning with two-control baselines: Random signal and signal absent. Psychophysiology, 1973, 10, 145-153.

Quinsey, V. L. Conditioned suppression with no CS-US contingency in the rat. Canadian Journal of Psychology. 1971, 25. 69-82.

Rescorla, R. A. Predictability and number of pairings in Pavlovian fear conditioning. Psychonomic Science, 1966. 4. 383-384.

Rescorla, R. A. Pavlovian conditioning and its proper control procedures. Psychological Review, 1967, 74, 71-80.

Rescorla, R. A. Conditioned inhibition of fear resulting from negative CS-US contingencies. Journal of Comparative and Physiological Psychology, 1969, 67, 504-509. (a)

Rescorla, R. A. Pavlovian conditioned inhibition. Psychological Review, 1969. 72. 77.94. (b)

Roberts, L. E., \& Young, R. Electrodermal responses are independent of movement during aversive conditioning in rats. but heart rate is not. Journal of Comparative and Physiological Psychology, 1971, 77, 495-512.

SIEGEL, S., \& DomJan, M. Backward conditioning as an inhibitory procedure. Learning and Motivation, 1971, 2. 1-11.

SIEGEL. S., \& Domuan. M. The inhibitory effect of backward conditioning as a function of the number of backward pairings. Bulletin of the Psychonomic Society, 1974, 4, 122-124.

SzWEJKowsKa, G. The transformation of differentiated inhibitory stimuli into positive conditioned stimuli. Acta Biologiae Experimentalis, 1959. 19.151-159.

(Received for publication August 26, 1976; revision accepted January 5, 1977.) 